\title{
Análise comparativa da velocidade do vento e da temperatura do ar, entre dados gerados por reanálises meteorológicas e dados observacionais na região de Minas Gerais
}

Comparative analysis of wind speed and air temperature, between data generated by meteorological reanalysis and observational data in the region of Minas Gerais

Mauren Lucila Marques de Morais Micalichen e Nelson Luís da Costa Dias

Universidade Federal do Paraná (UFPR), Brasil

\section{Resumo}

A utilização de fontes alternativas de dados meteorológicos tem se tornado cada vez mais usual, possibilitando uma avaliação de áreas com ausência de séries longas ou continuas de dados meteorológicos. Neste contexto, o principal objetivo deste estudo é avaliar o desempenho de séries de dados dos National Centers for Environmental Prediction / Nacional Center for Atmospheric Research (NCEP/NCAR) para o estado de Minas Gerais e verificar a possível utilização das mesmas na ausência de dados observados de temperatura do ar e velocidade do vento. As análises foram realizadas a partir da comparação entre dados observacionais de 17 estações meteorológicas e dados de reanálise dos modelos CFSR e CFSV2. A partir dos resultados das análises estatísticas, observa-se que os dados de reanálise de temperatura do ar apresentaram um bom desempenho na região de estudo. Porém os dados de velocidade do vento apontam uma correlação fraca. Esses resultados mostram que os dados de temperatura do ar dessas reanálises têm potencial para serem utilizados como fonte alternativa de dados. Sugere-se mais estudos em relação à utilização dos dados de velocidade do vento dessas reanálises.

Palavras-chave: Temperatura, velocidade do vento, estações meteorológicas, dados de reanálise, Minas Gerais.

\section{Abstract}

The use of alternative sources of meteorological data has become increasingly common, making it possible to evaluate areas with no long or continuous series of meteorological data. In this context, the main objective of this study is to evaluate the performance of data series from the National Centers for Environmental Prediction / National Center for Atmospheric Research (NCEP/NCAR) for the state of Minas Gerais and verify the possible use of them in the absence of data observations of air temperature and wind speed. The analyzes were performed by comparing observation data from 17 meteorological stations and reanalysis data of the CFSR and CFSV2 models. From the results of the statistical analysis, it is observed that the air temperature reanalysis data presented a good performance in the region of study. However, wind speed data show a weak correlation. These results show that the air temperature data from these reanalyses have the potential to be used as an alternative source of data. Further studies are suggested regarding the use of wind speed data from these reanalyses

Keywords: Temperature, wind speed, meteorological stations, reanalysis data, Minas Gerais. 


\section{Introdução}

Para diversos estudos atmosféricos são necessários dados observacionais com boa qualidade e disponibilidade. Mesmo sabendo da importância dessas observações, uma das grandes dificuldades para fazer estudos deste tipo ainda é a baixa quantidade e qualidade de informações de variáveis meteorológicas disponíveis. Algumas regiões possuem poucas estações meteorológicas para coleta desses dados, ou apresentam séries históricas com falhas de medição devido à falta de manutenção ou outros problemas técnicos. Diante desses problemas, alguns centros de previsão do tempo e clima disponibilizam produtos de reanálise, que são séries de dados meteorológicos obtidos através da assimilação e reanálise de dados observados em superfície (estações), aviões, navios, imagens de satélite, imagens de radar e radiossondagens em todo o planeta (Kalnay et al., 1996).

Porém, esses bancos de dados podem apresentar discrepâncias quando comparados com alguma fonte de dados observacionais, principalmente para regiões do planeta onde exista uma carência considerável de observações meteorológicas ou ainda onde a confiabilidade dos dados observados é questionável. Para uma melhor confiabilidade é necessário um estudo sobre a qualidade desses dados de reanálise. Um bom estudo de qualidade dos dados é uma etapa fundamental antes de se iniciar qualquer forma de uso dos dados de reanálise.

O presente trabalho tem por finalidade avaliar o desempenho das séries de dados do NCEP/NCAR para o estado de Minas Gerais e verificar a sua possível utilização na ausência de dados observados de velocidade do vento e temperatura do ar. Foi realizada uma análise comparativa a partir dos dados de reanálise e de dados coletados em estações automáticas.

\section{Dados e metodologia}

\section{$2.1 \quad$ Área de estudo}

O estado de Minas Gerais está localizado na Região Sudeste do Brasil entre as latitudes de $14^{\circ}$ e $22^{\circ}$ Sul (S) e as longitudes $39^{\circ}$ e $51^{\circ}$ Oeste (W). É o quarto estado brasileiro com maior área territorial $\left(586.528 \mathrm{~km}^{2}\right)$. Minas Gerais é inteiramente formado por planaltos, predominando o planalto Atlântico e também o planalto Central na porção noroeste, com planaltos sedimentares. A vegetação predominante é a do Cerrado. O clima varia desde o quente semiárido até o mesotérmico úmido (do Amarante et al., 2010).

\subsection{Dados de velocidade do vento e de temperatura do ar}

O conjunto de dados empregados neste estudo é constituído por séries provenientes da rede de estações meteorológicas de superfície da Companhia Energética de Minas Gerais (CEMIG). Para esta análise foram utilizados dados de velocidade do vento e temperatura do ar de 17 estações (Figura 1). Esta escolha deve-se ao fato de suas localizações estarem em destaque no atlas eólico de Minas Gerais (do Amarante et al., 2010), como áreas mais promissoras para empreendimentos eolioelétricos no estado. O período de estudo varia de estação para estação entre os anos de 1998 a 2015. Foram utilizados todos os dados disponíveis das estações meteorológicas escolhidas.

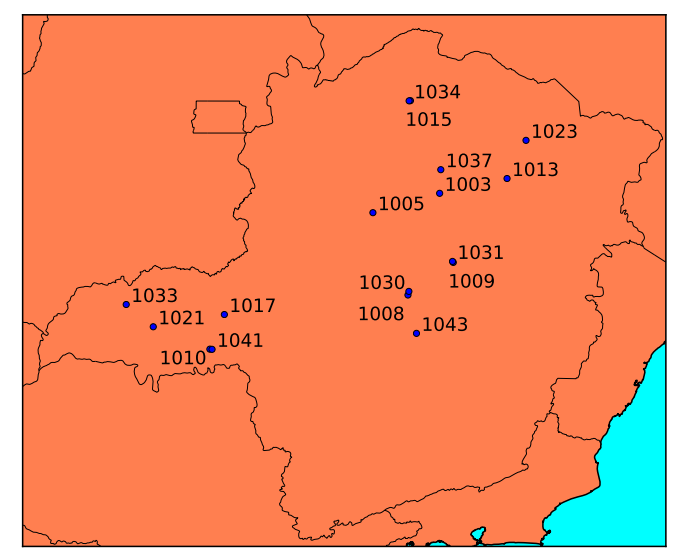

Figura 1: Mapa contendo a localização das 17 estações meteorológicas selecionadas. 
Além dos dados medidos nas estações meteorológicas, utilizamos os dados da velocidade do vento e da temperatura do ar dos produtos de reanálise dos NCEP/NCAR. Esses conjuntos de dados são séries provenientes de modelagem numérica atmosférica, possuem alta resolução horizontal (T382L64) de aproximadamente $38 \mathrm{~km}$ para o período entre 01/01/1979 e 31/03/2011, e são elaborados a partir do modelo Climate Forecast System Reanalysis (CFSR). Para os dados após 30 de março de 2011 até a presente dada, uma nova reanálise, denominada Climate Forecast System Version 2 (CFSV2), com resolução ainda melhor, de aproximadamente $22 \mathrm{~km}$, está sendo gerada e permite a extensão das séries até o presente (Saha et al., 2010). Essa nova reanálise foi utilizada para a comparação com os dados observacionais do perído de 01/04/2011-31/12/2015.

\subsection{Análise estatística}

A análise entre os dados de estações automáticas e os dados de reanálise do NCEP/NCAR foi feita com base nas médias horárias da temperatura do ar a 2 metros e da velocidade do vento a 10 metros de altura do solo.

A comparação dos dados meteorológicos observados com os dados de reanálise, para a avaliação do desempenho das séries de dados, foi realizada com cálculos estatísticos como: o viés, o erro médio absoluto (EMA), a raiz do erro quadrático médio (REQM), a variância (VAR) e o coeficiente de correlação $(r)$. As expressões empregadas nesta avaliação foram adaptadas de Wilks (2006):

$$
\begin{aligned}
V I E S S & =\frac{1}{n} \sum_{i=1}^{n}\left(x_{r}-x_{o}\right) \\
E M A & =\frac{1}{n} \sum_{i=1}^{n}\left|x_{r}-x_{o}\right| \\
R E Q M & =\sqrt{\frac{1}{n} \sum_{i=1}^{n}\left(x_{r}-x_{o}\right)^{2}} \\
V A R & =\sqrt{\frac{1}{n-1} \sum_{i=1}^{n}\left(x_{i}-\bar{x}\right)^{2}} \\
r & =\frac{c_{x_{r} x_{o}}}{s_{x_{r}} s_{x_{o}}}
\end{aligned}
$$

Nas equações (1) a (5), $n$ é o número de amostras, $x_{r}$ é o dado da reanálise, $x_{o}$ é o dado das observações, $\bar{x}$ é a média dos dados, $c_{x_{r} x_{o}}$ é a covariância entre os pares de dados da reanálise e os dados observados, $s_{x_{r}}$ é o desvio padrão dos dados da reanálise e $s_{x_{o}}$ é o desvio padrão dos dados das observações.

Ressalta-se que alguns desses índices foram utilizados em estudos semelhantes de comparação entre dados observados e simulados realizados por Mariano et al. (2017), Stüker et al. (2016), Kaiser-Weiss et al. (2015), Saldanha et al. (2015), Vieira et al. (2006) entre outros.

\section{Resultados e Discussão}

A primeira etapa da análise foi referente à quantidade de anos em que as estações estiveram ativas e quais as medições realizadas em cada uma delas. Com isso foram selecionados todos os dados a serem utilizados.

Os gráficos da Figura 2 e da Figura 3, mostram a temperatura do ar e a velocidade do vento da estação 1031 para duas estações do ano em um período de duas semanas. Visualmente já é possível observar que a temperatura do ar parece acompanhar muito bem os dados de reanálise. Porém, a velocidade do vento não segue o mesmo padrão.

Após a análise visual das séries horárias, foram calculados os índices estatísticos para todo o período disponível de cada estação, portanto não necessariamente para um período comum. Na Tabela 1 e Tabela 2 encontram-se os valores obtidos nos cálculos estatísticos feitos para a análise da temperatura do ar e da velocidade do vento, respectivamente.

Os índices estatísticos apresentados na Tabela 1 indicam que os dados de temperatura do ar apresentaram o menor EMA para a estação Diamantina $\left(1,8045^{\circ} \mathrm{C}\right)$ e o maior EMA para Salinas $\left(2,6139{ }^{\circ} \mathrm{C}\right)$. Já para a REQM, o mínimo $\left(2,4452^{\circ} \mathrm{C}\right)$ se deu para Diamantina e o máximo $\left(3,2104^{\circ} \mathrm{C}\right)$ para Salinas. Quanto ao viés, a grande maioria dos valores apresentados são negativos indicando uma subestimação dos dados simulados em relação aos observados, os únicos valores positivos ocorreram para a estações de Diamantina $\left(0.9324^{\circ} \mathrm{C}\right)$, Uberaba $\left(0,6521^{\circ} \mathrm{C}\right)$ e Prata $\left(0,1334^{\circ} \mathrm{C}\right)$.

Para os dados de velocidade do vento, verifica-se na Tabela 2 que os menores valores de EMA e REQM foram $0,8666 \mathrm{~m} / \mathrm{s}$ e $1,0719 \mathrm{~m} / \mathrm{s}$, respectivamente, e ocorreram nas localidades de Curvelo e Igicatu. Já os maiores valores de EMA e REQM foram 1,3844 m/s e 1,7095 m/s e ocorreram em SE Montes Claros e Diamantina, respectivamente. O viés apresentou $47 \%$ dos 
Temperatura estação 1031 (01 a 14 de janeiro - 2009)

Temperatura estação 1031 (01 a 14 de julho - 2009)

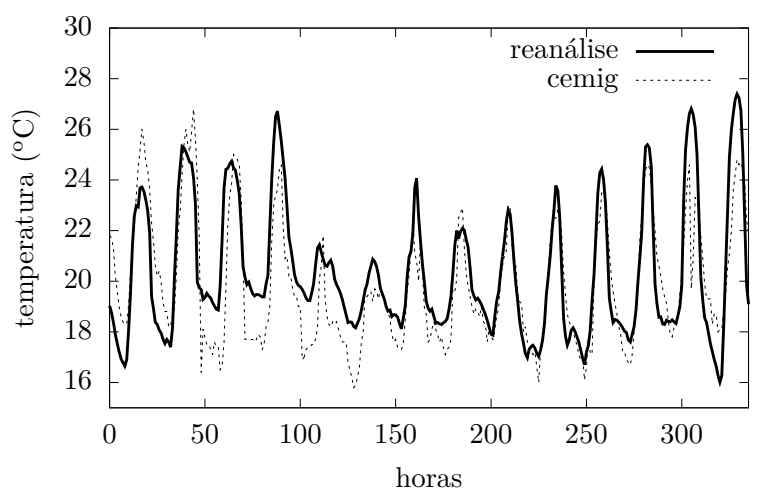

Figura 2: Gráfico comparativo de temperatura para a estação 1031 - Reanálise x Cemig.

Velocidade do vento estação 1031 (01 a 14 de janeiro - 2009)

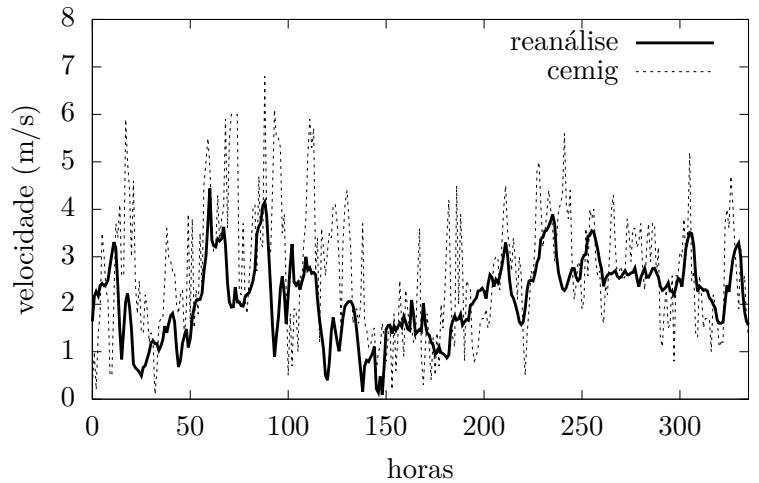

Velocidade do vento estação 1031 (01 a 14 de julho - 2009)

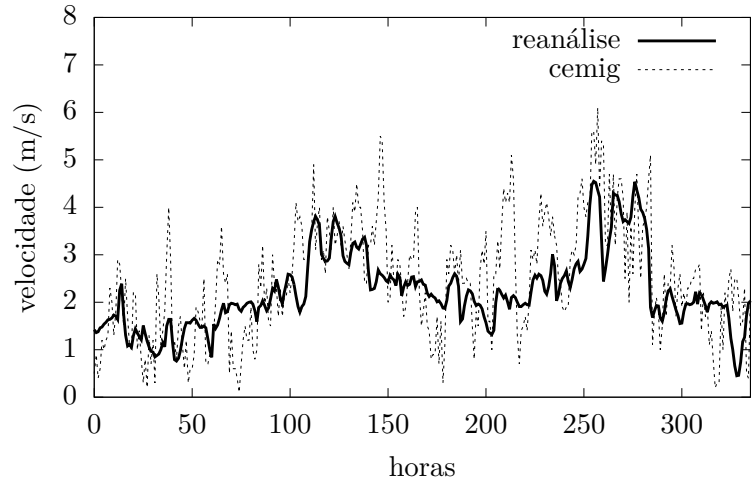

Figura 3: Gráfico comparativo de velocidade do vento para a estação 1031 - Reanálise x Cemig.

Tabela 1: Estatísticas com dados de temperatura $\left({ }^{\circ} \mathrm{C}\right)$

\begin{tabular}{rcccccc}
\hline Estação & Viés & EMA & REMQ & Coef. Correlação & Var. Reanálise & Var. Cemig \\
\hline Bocaiúva 1003 & $-0,6155$ & 1,8997 & 2,4744 & 0,8574 & 21,6658 & 15,3427 \\
Buritizeiro 1005 & $-1,0110$ & 2,4025 & 3,0299 & 0,8299 & 25,0094 & 22,6713 \\
Curvelo 1008 & $-0,6345$ & 2,0177 & 2,6005 & 0,8578 & 24,0701 & 18,1021 \\
Diamantina 1009 & 0,9324 & 1,8045 & 2,4452 & 0,8560 & 18,9019 & 12,1160 \\
Uberaba 1010 & 0,6521 & 2,1719 & 2,8889 & 0,8465 & 27,0227 & 24,1283 \\
Igicatu 1013 & $-0,2070$ & 2,0498 & 2,6411 & 0,8522 & 24,9651 & 15,5865 \\
Januária 1015 & $-0,6314$ & 2,0943 & 2,7535 & 0,8548 & 24,2409 & 25,1838 \\
Nova Ponte 1017 & $-1,0773$ & 2,2556 & 2,8301 & 0,8488 & 20,5462 & 23,9992 \\
Prata 1021 & 0,1334 & 2,2703 & 3,0431 & 0,8304 & 29,3509 & 23,6035 \\
Salinas 1023 & $-1,8390$ & 2,6139 & 3,2104 & 0,8422 & 23,3352 & 19,7572 \\
SE Curvelo 1030 & $-0,7415$ & 2,1103 & 2,6970 & 0,8507 & 24,3423 & 17,6064 \\
SE Diamantina 1031 & $-0,1195$ & 1,8085 & 2,3647 & 0,8290 & 17,7463 & 11,0767 \\
SE Ituiutaba 1033 & $-1,1107$ & 2,1036 & 2,7322 & 0,8630 & 21,0413 & 23,8732 \\
SE Januária 1034 & $-1,7984$ & 2,5203 & 3,1807 & 0,8416 & 23,5972 & 16,9039 \\
SE Montes Claros 1037 & $-1,8690$ & 2,3720 & 2,9885 & 0,8755 & 20,3403 & 22,8320 \\
SE Uberaba 1041 & $-0,5330$ & 1,9630 & 2,6231 & 0,8406 & 22,3987 & 17,0619 \\
Sete Lagoas 1043 & $-1,6948$ & 2,3706 & 2,9734 & 0,8319 & 19,3839 & 13,6650 \\
\hline
\end{tabular}


Tabela 2: Estatísticas com dados de velocidade do vento $(\mathrm{m} / \mathrm{s})$

\begin{tabular}{rcccccc}
\hline Estação & Viés & EMA & REMQ & Coef. Correlação & Var. Reanálise & Var. Cemig \\
\hline Bocaiúva 1003 & $-0,3028$ & 1,2562 & 1,6945 & 0,1415 & 1,1086 & 2,1033 \\
Buritizeiro 1005 & $-0,1902$ & 1,1000 & 1,4179 & 0,2833 & 0,7750 & 1,8838 \\
Curvelo 1008 & 0,1059 & 0,8666 & 1,1033 & 0,3847 & 0,8841 & 1,0706 \\
Diamantina 1009 & $-1,1062$ & 1,3255 & 1,7095 & 0,4820 & 0,6932 & 2,1945 \\
Uberaba 1010 & $-0,3994$ & 1,0088 & 1,3171 & 0,4911 & 1,0365 & 1,9268 \\
Igicatu 1013 & $-0,4258$ & 0,8580 & 1,0719 & 0,3699 & 0,7924 & 0,7428 \\
Januária 1015 & 0,6812 & 1,1116 & 1,3793 & 0,3493 & 0,9354 & 1,2624 \\
Nova Ponte 1017 & 0,3005 & 1,2935 & 1,6307 & 0,3302 & 1,1486 & 2,5510 \\
Prata 1021 & $-0,7142$ & 1,2164 & 1,5965 & 0,4824 & 0,9582 & 2,6050 \\
Salinas 1023 & 0,2484 & 1,0444 & 1,2894 & 0,4270 & 0,8376 & 1,8167 \\
SE Curvelo 1030 & $-0,4867$ & 1,1502 & 1,4786 & 0,4259 & 0,8264 & 2,2964 \\
SE Diamantina 1031 & $-0,1997$ & 0,9274 & 1,1997 & 0,4104 & 0,7714 & 1,5155 \\
SE Ituiutaba 1033 & 0,2513 & 1,0205 & 1,2975 & 0,3977 & 0,9447 & 1,6768 \\
SE Januária 1034 & 0,0271 & 0,9946 & 1,2725 & 0,4190 & 1,0140 & 1,7070 \\
SE Montes Claros 1037 & 1,2003 & 1,3844 & 1,6741 & 0,3943 & 1,1075 & 1,1408 \\
SE Uberaba 1041 & 0,0963 & 0,8977 & 1,1559 & 0,4654 & 1,0263 & 1,4268 \\
Sete Lagoas 1043 & 0,1638 & 0,9831 & 1,2536 & 0,3373 & 0,8696 & 1,4263 \\
\hline
\end{tabular}

dados com valores negativos, o que indica uma subestimação dos dados de reanálise em relação aos observados, 53\% dos dados apresentaram valores positivos mostrando que houve uma superestimação.

Em relação ao coeficiente de correlação entre os dados observados e os das reanálises, para a temperatura do ar o coeficiente de correlação médio foi de 0,8475 , indicando uma forte correlação com os dados observados. Já os resultados da correlação para a velocidade do vento se mostraram inferiores apresentando um coeficiente médio de 0,3877 , mostrando uma correlação fraca na média. $47 \%$ dos dados de vento apresentaram uma correlaçao moderada, superior a $0,4 \mathrm{~m} / \mathrm{s}$.

O melhor resultado em relação ao coeficiente de correlação da temperatura do ar está localizado na SE Montes Claros $(0,8755)$ no norte de MG. Já para a velocidade do vento o melhor resultado está localizado na SE Uberaba $(0,4911)$ na mesorregião do Triângulo Mineiro e Alto Paranaíba de MG. Os coeficientes com piores resultados foram na SE Diamantina com correlação de 0.8290 para a temperatura e Bocaiúva com correlação de 0,1415 para velocidade do vento.

De maneira geral, os dados de reanálise em relação aos dados das estações indicaram uma maior variação média da temperatura. Já para velocidade do vento a variação das estações da rede Cemig foi maior, ou seja, os dados de reanálises não conseguiram acompanhar as variações dos dados observados.

Considerando o conjunto de resultados apresentados acima, vê-se claramente que a reanálise da temperatura do ar obteve desempenho melhor comparada com a reanálise da velocidade vento.

\section{Conclusões}

O principal objetivo desta análise foi avaliar o desempenho das séries de dados dos NCEP/NCAR para o estado de Minas Gerais e verificar as suas possíveis utilizações na ausência de dados observados de velocidade do vento e temperatura do ar.

Os resultados da análise dos dados de temperatura do ar indicaram que os dados de reanálise dos modelos CFSR/CFSV2 apresentaram um bom desempenho em relação aos dados observados, com coeficiente de correlação médio em torno de 0,8475 . Portanto conclui-se que esses dados apresentam potencial para serem utilizados como fonte alternativa em locais com ausência de estações ou de séries longas de dados meteorológicos.

Já os dados de velocidade do vento das reanálises não reproduziram a variabilidade apresentada pelas estações automáticas. O coeficiente de correlação médio encontrado foi de 0,3877 .

Os estudos feitos demonstram a importância na escolha apropriada da fonte de longo prazo para realização de estudos meteorológicos.

Apenas com os resultados provenientes desse estudo não se pode tirar conclusões definitivas sobre essas fontes de dados; para isso, estudos com mais estações meteorológicas em diferentes localidades e com diferentes períodos de dados devem ser realizados.

\section{Referências}

do Amarante, O. A. C., de Jesus Lima da Silva, F., de Andrade, P. E. P. (2010). Atlas eólico - minas gerais. Recuperado em http://www.cemig.com.br/pt-br/A_Cemig_e_o_Futuro/inovacao/Alternativas_ Energeticas/Documents/atlas\%20eolico\%20MG.pdf Acesso em: 23/06/2016.. 
Kaiser-Weiss, A. K., Kaspar, F., Heene, V., Borsche, M., Tan, D. G. H., Poli, P., Obregon, A., Gregow, H. (2015). Comparison of regional and global reanalysis near-surface winds with station observations over germany. Advances in Science and Research.

Kalnay, E., Kanamitsu, M., Kistler, R., Collins, W., Deaven, D., L. Gandin, M. I., Saha, S., White, G., Woollen, J., Zhu, Y., Leetmaa, A., Reynolds, R., Chelliah, M., Ebisuzaki, W., Higgins, W., Janowiak, J., Mo, K. C., Ropelewski, C., Wang, J., Jenne, R., Joseph, D. (1996). The ncep/ncar 40-year reanalysis project. American Meteorological Society.

Mariano, E. B., Cavalcanti, E. P., Beserra, E. A. (2017). Análise comparativa da velocidade do vento simulado pelo brams com dados observados e de reanálises. Revista Brasileira de Meteorologia.

Saha, S., Moorthi, S., Pan, H. L., Wu, X., Wang, J., Nadiga, S., Tripp, P., Kistler, R., Woollen, J., Behringer, D., Liu, H., Stokes, D., Grumbine, R., Gayno, G., Wang, J., Hou, Y. T., Chuang, H. Y., Juang, H. M. H., Sela, J., Iredell, M., Treadon, R., Kleist, D., Delst, P. V., Keyser, D., Derber, J., Ek, M., Meng, J., Wei, H., Yang, R., Lord, S., Dool, H. V. D., Kumar, A., Wang, W., Long, C., Chelliah, M., Xue, Y., Huang, B., Schemm, J. K., Ebisuzaki, W., Lin, R., Xie, P., Chen, M., Zhou, S., Higgins, W., Zou, C. Z., Liu, Q., Chen, Y., Han, Y., Cucurull, L., Reynolds, R. W., Rutledge, G., Goldberg, M. (2010). The ncep climate forecast system reanalysis. Bulletin of the American Meteorological Society, 91(8), 1015-1057, http://dx.doi.org/10.1175/2010BAMS3001.1.

Saldanha, C. B., Radin, B., Cardoso, M. A. G., Rippel, M. L., Fonseca, L. L. D., Rodriguez, F. (2015). Comparação dos dados de precipitação gerados pelo gpcp vs observados para o estado do rio grande do sul. Revista Brasileira de Meteorologia.

Stüker, E., Schuster, C. H., Schuster, J. J., Santos, D. C., Medeiros, L. E., Costa, F. D., e Franciano Scremin Puhales, G. D. (2016). Comparação entre os dados de vento das reanálises meteorológicas era-interim e cfsr com os dados das estações automáticas do inmet no rio grande do sul. Ciência e Natura.

Vieira, C. F. A., dos Santos, C. C., de Lima, F. J. L., Magalhães, R. A., da Silva, E. M. (2006). Avaliação dos dados de vento gerados no projeto reanalysis do ncep/ncar para futuras aplicações no cálculo do potencial eólico em regiões do estado do ceará. Rev Tecnol Fortaleza, 27, 190-194.

Wilks, D. S. (2006). Statistical Methods in the Atmospheric Sciences, Internacional Geophysics Series, vol 91, $2^{\circ}$ edn. Academic Press.

Mauren Lucila Marques de Morais Micalichen

Universidade Federal do Paraná (UFPR), Brasil E-mail: mauren_morais@hotmail.com

Nelson Luís da Costa Dias

Universidade Federal do Paraná (UFPR), Brasil E-mail: nelsonluisdias@gmail.com 